\title{
Sexual Dimorphism of Craniodental Morphology in the Raccoon Dog Nyctereutes procyonoides from South Korea
}

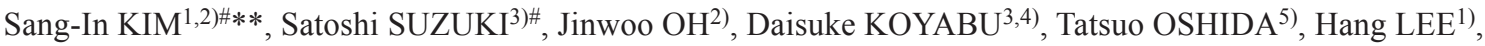 \\ Mi-Sook MIN ${ }^{1)}$ and Junpei KIMURA ${ }^{2) *}$ \\ ${ }^{1)}$ Conservation Genome Resource Bank for Korean Wildlife (CGRB), Research Institute for Veterinary Science, College of Veterinary \\ Medicine, Seoul National University, Seoul 151-724, Korea \\ ${ }^{2)}$ Department of Anatomy and Cell Biology, College of Veterinary Medicine, Seoul National University, Seoul 151-742, Korea \\ ${ }^{3)}$ The Kyoto University Museum, Kyoto University, Sakyo, Kyoto 606-8501, Japan \\ 4) Palaeontological Institute and Museum, University of Zurich, Zurich 8006, Switzerland \\ ${ }^{5)}$ Laboratory of Wildlife Biology, Obihiro University of Agriculture and Veterinary Medicine, Obihiro 080-8555, Japan
}

(Received 23 June 2012/Accepted 21 July 2012/Published online in J-STAGE 3 August 2012)

ABSTRACT. We examined sexual dimorphism in the craniodental traits of the raccoon dog Nyctereutes procyonoides from South Korea. Univariate comparisons of skull (cranium and mandible) and dental measurements revealed a small extent of sexual dimorphism in some measurements. The most indicative dimorphic measurements were the breadths of the upper and lower canines which were around $8 \%$ larger in male specimens on average. On the other hand, multivariate analyses using only skull traits showed slightly a clearer separation between sexes than those using only dental ones. This discrepancy may be derived from a higher variability in dental traits than in those of the skull. In conclusion, sexual dimorphism within $N$. procyonoides of South Korea is present, but was not so pronounced as for other local populations. However, measurements showing significant sexual dimorphism varied between different localities. This suggests that the selective forces acting upon craniodental morphology of each sex vary between populations of the species.

KEY WORDS: craniodental morphology, Nyctereutes procyonoides koreensis, raccoon dog, sexual dimorphism.

doi: 10.1292/jvms.12-0281; J. Vet. Med. Sci. 74(12): 1609-1616, 2012

Sexual size dimorphism (SSD) is a well-recognized phenomenon, which is observed in many taxa of mammals. Especially, most species in the order Carnivora show clear SSD [4, 12, 14, 22]. Therefore, this taxon has been regarded as a suitable model group to study sexual differentiation in morphology [4]. However, several species of Canidae exhibit exceptionally low to negligible SSD. In the raccoon dog Nyctereutes procyonoides, SSD has been studied by several authors [7, 10, 13, 16]. Nyctereutes procyonoides has originally held a wide distribution throughout East Asia, however, it has widely established itself throughout Eastern Europe, following introduction to the continent by man. The species shows remarkable geographic variation in the skull and teeth among both native and introduced populations $[1,5,10,17]$. The sexual dimorphism is reported to be relatively small in raccoon dogs $[5,7,10,16]$, nevertheless, the degree of sexual dimorphism varies between its local populations $[5,16]$. To elucidate the factors which have influenced sexual dimorphism within raccoon dogs, an assessment of the geographic variation in sexual dimorphism

\footnotetext{
*Correspondence to: Kimura, J., Department of Anatomy and Cell Biology, College of Veterinary Medicine, Seoul National University, Seoul 151-742, Korea.

e-mail:kimura@snu.ac.kr

**PRESENT ADDRESS: Laboratory of Wildlife Biology, Obihiro University of Agriculture and Veterinary Medicine, Obihiro 080-8555, Japan.

\# The first two authors contributed equally to this study.

(C)2012 The Japanese Society of Veterinary Science
}

should be helpful.

In $N$. procyonoides, there is only one study which has compared the regional differences in sexual dimorphism as noted above [16], though further authors have studied the dimorphism within one region $[5,6]$. In this study, we examined sexual dimorphism of the craniodental traits among South Korean specimens, which are regarded as a distinct subspecies, $N$. p. koreensis [15]. This report is the first study to examine the sexual dimorphism of $N$. p. koreensis in South Korea, and should contribute to our understanding of factors relating to the evolution of sexual dimorphism.

\section{MATERIALS AND METHODS}

We examined sexual dimorphism using craniodental measurements of 63 individuals of $N$. p. koreensis from South Korea (male: 37, female: 26). Only adult specimens of which the lower third molar (m3) is fully erupted, were used in order to eliminate age related variation. By using 17 cranial, 7 mandibular and 30 dental measurements (Table 1; Fig. 1), we conducted univariate and multivariate comparisons. These measurement techniques are those from Haba et al. [5], though the heights of the upper and lower canines $(\mathrm{CH}$ and $\mathrm{cH})$ and the SIZE, the skull length independent of rostrum length (CBL-RL) were excluded. In univariate treatments, we compared the mean and variance values of each measurement by Welch's $t$-test and $F$-test, respectively. As multivariate treatments, we conducted principal component analysis (PCA) based on variance-covariance matrix and canonical variate analysis (CVA) using natural log- 

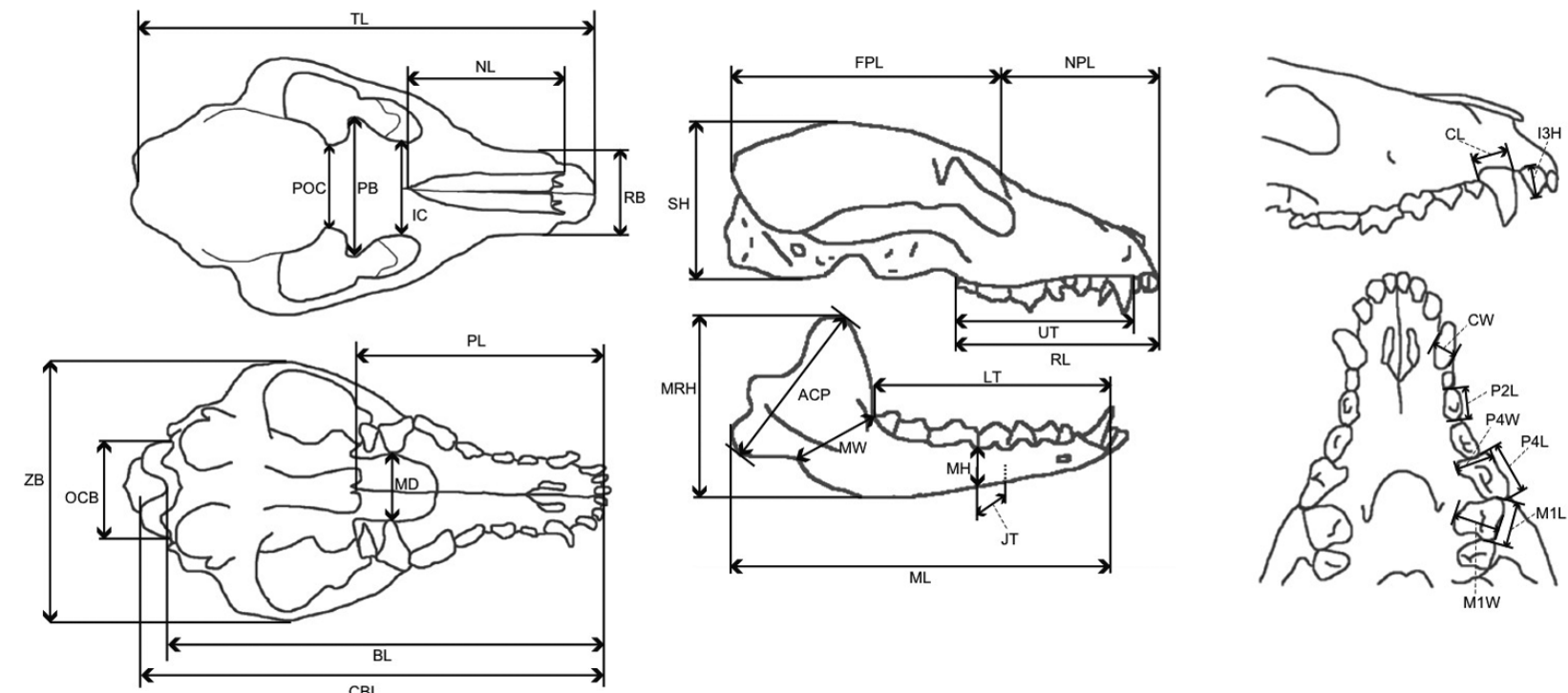

Fig. 1. Skull and tooth measurements of the raccoon dog used in this study.

transformed values of skull (cranium and mandible) (sPCA and sCVA) and dental measurements (tPCA and tCVA) separately. For these analyses, we used specimens without missing values (skull; male: 36 , female: 25 , teeth; male: 35 , female: 24). All statistical analyses were conducted with the software R.2.13.2 [20].

\section{RESULTS}

Univariate analyses: Mean values were different between sexes in 5 variables (POC, JT, cW, m3L and CW) (Table 2). Canine measurements $(\mathrm{cW}$ and $\mathrm{CW}$ ) and $\mathrm{POC}$ were larger in male specimens, while JT and $\mathrm{m} 3 \mathrm{~L}$ were larger in females. Differences of variance were observed in 4 variables (RL, $\mathrm{MW}, \mathrm{p} 3 \mathrm{~L}$ and $\mathrm{I} 1 \mathrm{H})$.

Multivariate analyses: In the sPCA, the first 3 axes (sPC1, sPC2 and sPC3) explained 36.6, 12.5, and $11.3 \%$ of the total variation, respectively (Table 3). Factor loadings for $\mathrm{sPC1}$ were all positive except for POC, and therefore this variable indicated size variation and POC is not correlated with overall skull size. Factor loadings for $\mathrm{SPC} 2$ were large in POC (0.79), PB (0.62), IC (0.48) and MH (-0.45), and those for SPC3 were large in MW (0.69) and JT (-0.47). Scores of $\mathrm{SPC} 1$ were not different between the sexes $(P=0.19)$, and variance of the score was marginally significantly larger in female $(P=0.077)$ (Fig. 2A). On the other hand, sPC2 scores were larger in male $(P=0.0052)$, although the ranges overlapped to a high degree, and variances were not significantly different $(P=0.35)$.

In the tPCA, the first 3 axes accounted for 32.1, 15.4 and $10.0 \%$ of the total variation, respectively (Table 4 ). Factor loadings for tPC1 were larger in incisors. Those for tPC2 were large in canines, some premolars, $\mathrm{m} 1$ and $\mathrm{M} 1$, and those for $\mathrm{tPC} 3$ were larger in $\mathrm{m} 3$, followed by canine lengths (0.40 to 0.43$)$. Cumulatively, the first 7 axes accounted for
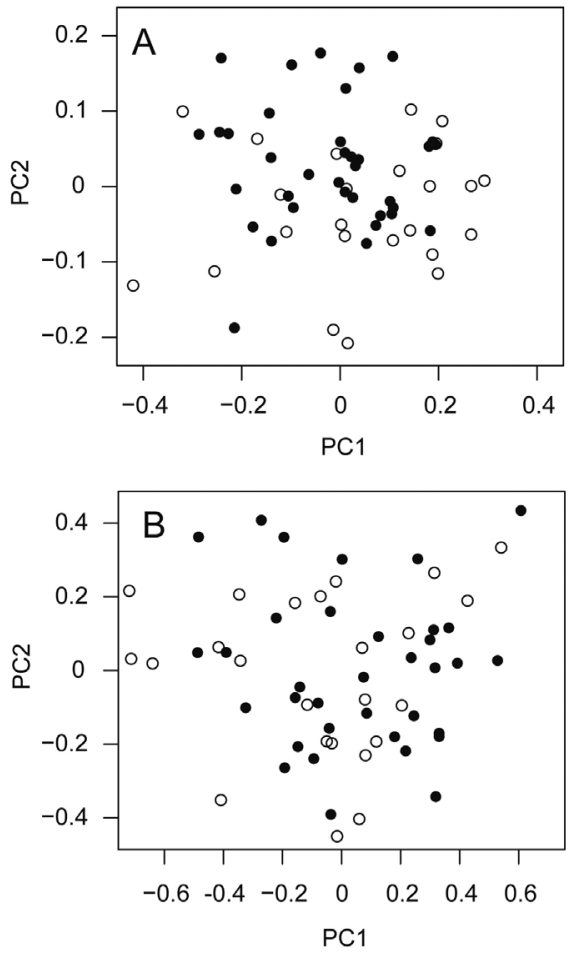

Fig. 2. Two dimensional plots of the first and second principal component axes in skull measurements (A) and dental measurements (B). Open circles: male, closed circles: female.

more than $80 \%$ in total. Each of these axes did not show significant difference of either mean or variance between sexes except for tPC4 which explains $8.0 \%$ of the total variation showing significant sexual difference of means $(P=0.026)$ 
Table 1. List of skull and tooth measurements of the raccoon dog used in this study

\begin{tabular}{|c|c|c|}
\hline Category & Acronym & Measurement \\
\hline \multirow[t]{17}{*}{ Cranium } & NPL & Nasal + premaxillary length \\
\hline & NL & Nasal length \\
\hline & FPL & Frontal + parietal length \\
\hline & SH & Skull height \\
\hline & BL & Basal length \\
\hline & $\mathrm{RB}$ & Rostrum breadth \\
\hline & POC & Postorbital constriction \\
\hline & IC & Interorbital constriction \\
\hline & $\mathrm{PB}$ & Postorbital breadth \\
\hline & OCB & Occipital condyle breadth \\
\hline & ZB & Zygomatic breadth \\
\hline & $\mathrm{TL}$ & Total length \\
\hline & PL & Palatal length \\
\hline & MD & Distance between first upper molars \\
\hline & CBL & Condylobasal length \\
\hline & UT & Length of the upper toothrow \\
\hline & RL & Rostrum length \\
\hline \multirow[t]{7}{*}{ Mandible } & MRH & Height of the mandibular ramus \\
\hline & $\mathrm{ACP}$ & $\begin{array}{l}\text { Length from angular process to coronoid } \\
\text { process }\end{array}$ \\
\hline & LT & Length of the lower toothrow \\
\hline & JT & Jaw thickness \\
\hline & MH & Mandibular height \\
\hline & ML & Mandible length \\
\hline & MW & Mandible width \\
\hline \multirow[t]{30}{*}{ Tooth } & $\mathrm{i} 1 \mathrm{H}$ & Height of the lower first incisor \\
\hline & $\mathrm{i} 2 \mathrm{H}$ & Height of the lower second incisor \\
\hline & $\mathrm{i} 3 \mathrm{H}$ & Height of the lower third incisor \\
\hline & $\mathrm{cL}$ & Length of the lower canine \\
\hline & $\mathrm{cW}$ & Width of the lower canine \\
\hline & p1L & Length of the lower first premolar \\
\hline & $\mathrm{p} 2 \mathrm{~L}$ & Length of the lower second premolar \\
\hline & $\mathrm{p} 3 \mathrm{~L}$ & Length of the lower third premolar \\
\hline & $\mathrm{p} 4 \mathrm{~L}$ & Length of the lower fourth premolar \\
\hline & $\mathrm{p} 4 \mathrm{~W}$ & Width of the lower fourth premolar \\
\hline & $\mathrm{m} 1 \mathrm{~L}$ & Length of the lower first molar \\
\hline & $\mathrm{m} 1 \mathrm{~W}$ & Width of the lower first molar \\
\hline & $\mathrm{m} 2 \mathrm{~L}$ & Length of the lower second molar \\
\hline & $\mathrm{m} 2 \mathrm{~W}$ & Width of the lower second molar \\
\hline & $\mathrm{m} 3 \mathrm{~L}$ & Length of the lower third molar \\
\hline & $\mathrm{m} 3 \mathrm{~W}$ & Width of the lower third molar \\
\hline & $\mathrm{I} 1 \mathrm{H}$ & Height of the upper first incisor \\
\hline & $\mathrm{I} 2 \mathrm{H}$ & Height of the upper second incisor \\
\hline & $\mathrm{I} 3 \mathrm{H}$ & Height of the upper third incisor \\
\hline & CL & Length of the upper canine \\
\hline & $\mathrm{CW}$ & Width of the upper canine \\
\hline & P1L & Length of the upper first premolar \\
\hline & $\mathrm{P} 2 \mathrm{~L}$ & Length of the upper second premolar \\
\hline & P3L & Length of the upper third premolar \\
\hline & P4L & Length of the upper fourth premolar \\
\hline & $\mathrm{P} 4 \mathrm{~W}$ & Width of the upper fourth premolar \\
\hline & M1L & Length of the upper first molar \\
\hline & M1W & Width of the upper first molar \\
\hline & $\mathrm{M} 2 \mathrm{~L}$ & Length of the upper second molar \\
\hline & $\mathrm{M} 2 \mathrm{~W}$ & Width of the upper second molar \\
\hline
\end{tabular}
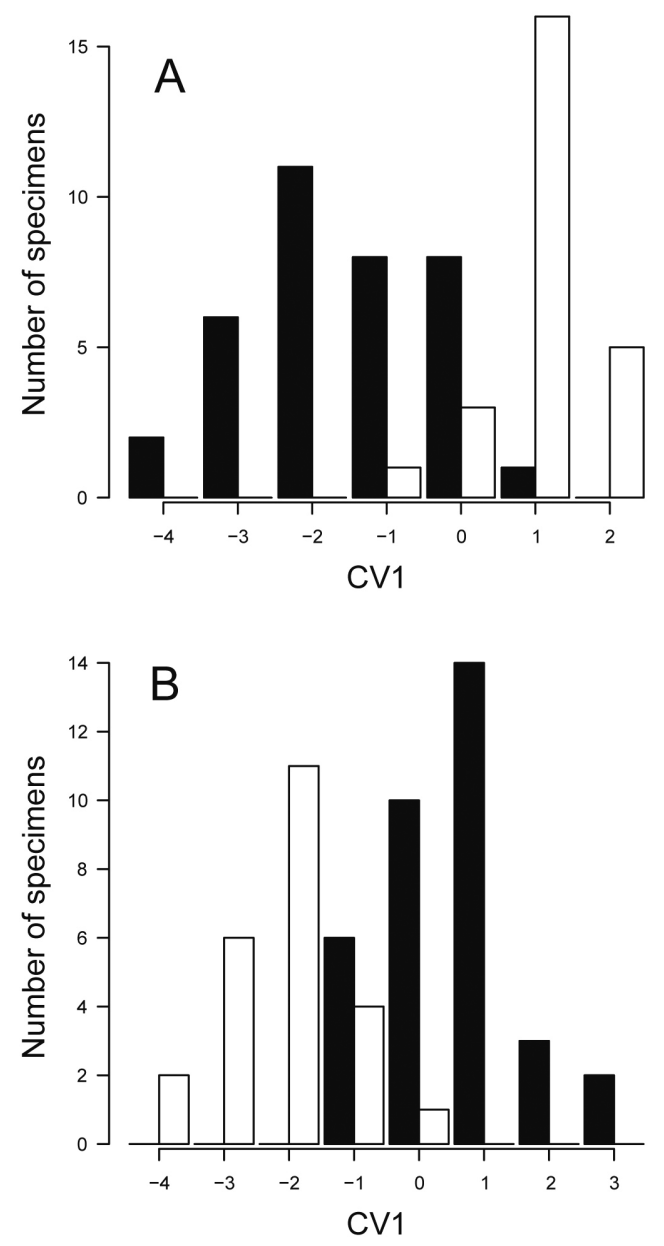

Fig. 3. Frequency distribution of CV1 scores by sCVA (A) and tCVA (B). Black: male, white: female.

(Fig. 2B).

By the CVA using skull and dental variables, 93.4 and $88.1 \%$ of specimens were correctly classified into each sex, respectively (Fig. 3). Standardized canonical coefficients for CV1 were large in TL (1.75), MRH (-1.53), MH (1.20), RL $(-1.14)$ and BL $(-1.13)$ in sCVA and large in CW (1.02), m1L (0.96), M1L (-0.92) and P4W (-0.85) in tCVA.

\section{DISCUSSION}

In the present study, we found that sexual dimorphism of craniodental traits of $N$. p. koreensis in South Korea is not strongly pronounced. Similarly, several previous studies have shown that sexual dimorphism of craniodental traits in this species is quite low, or possibly absent in other regions $[5,7,10,13,16]$. Although both the current work and that of Haba et al. [5] which studied sexual dimorphism in 2 populations (Hokkaido and Honshu), similarly detected significant sexual dimorphism in several canine variables, those traits which showed sexual dimorphism were different between these 3 populations. The most dimorphic variables 
Table 2. Descriptive statistics and results of comparison of skull and dental variables in each sex of the raccoon dog from South Korea

\begin{tabular}{|c|c|c|c|c|c|c|c|c|c|c|}
\hline \multirow{2}{*}{ Variable } & \multirow{2}{*}{ Sex } & \multirow{2}{*}{$\mathrm{N}$} & \multirow{2}{*}{ Mean } & \multirow{2}{*}{ Ratio (\%) } & \multicolumn{2}{|c|}{ Welch's $t$-test } & \multirow{2}{*}{ Variance } & \multirow{2}{*}{ Ratio (\%) } & \multicolumn{2}{|c|}{$F$-test } \\
\hline & & & & & $t$ & $P$ & & & $F$ & $P$ \\
\hline \multirow[t]{2}{*}{ NPL } & Male & 37 & 54.68 & 98.78 & -0.864 & 0.391 & 8.72 & 89.19 & 0.892 & 0.740 \\
\hline & Female & 26 & 55.35 & & & & 9.78 & & & \\
\hline \multirow[t]{2}{*}{ NL } & Male & 37 & 43.99 & 98.59 & -0.845 & 0.402 & 7.04 & 75.14 & 0.751 & 0.425 \\
\hline & Female & 26 & 44.62 & & & & 9.37 & & & \\
\hline \multirow[t]{2}{*}{ FPL } & Male & 37 & 68.54 & 99.29 & -0.640 & 0.525 & 8.43 & 91.31 & 0.913 & 0.789 \\
\hline & Female & 26 & 69.03 & & & & 9.23 & & & \\
\hline \multirow[t]{2}{*}{$\mathrm{SH}$} & Male & 37 & 43.79 & 100.50 & 0.473 & 0.639 & 2.33 & 60.19 & 0.602 & 0.160 \\
\hline & Female & 26 & 43.57 & & & & 3.87 & & & \\
\hline \multirow[t]{2}{*}{$\mathrm{BL}$} & Male & 37 & 110.47 & 100.36 & 0.410 & 0.683 & 11.68 & 70.18 & 0.702 & 0.325 \\
\hline & Female & 26 & 110.07 & & & & 16.64 & & & \\
\hline \multirow[t]{2}{*}{$\mathrm{RB}$} & Male & 37 & 22.22 & 100.54 & 0.510 & 0.613 & 0.66 & 68.74 & 0.687 & 0.298 \\
\hline & Female & 26 & 22.10 & & & & 0.96 & & & \\
\hline \multirow[t]{2}{*}{ POC } & Male & 37 & 20.42 & 104.44 & 2.245 & 0.029 & 2.15 & 90.63 & 0.906 & 0.773 \\
\hline & Female & 26 & 19.55 & & & & 2.37 & & & \\
\hline IC & Male & 37 & 24.04 & 100.00 & 0.000 & 1.000 & 1.12 & 56.65 & 0.567 & 0.117 \\
\hline & Female & 26 & 24.04 & & & & 1.97 & & & \\
\hline PB & Male & 37 & 33.37 & 101.77 & 1.261 & 0.213 & 2.67 & 74.27 & 0.743 & 0.407 \\
\hline & Female & 26 & 32.79 & & & & 3.60 & & & \\
\hline $\mathrm{OCB}$ & Male & 37 & 22.35 & 99.07 & -0.823 & 0.415 & 0.71 & 59.65 & 0.597 & 0.153 \\
\hline & Female & 26 & 22.56 & & & & 1.18 & & & \\
\hline ZB & Male & 36 & 67.03 & 101.03 & 0.932 & 0.356 & 6.54 & 70.73 & 0.707 & 0.340 \\
\hline & Female & 26 & 66.35 & & & & 9.24 & & & \\
\hline $\mathrm{TL}$ & Male & 37 & 118.77 & 99.35 & -0.744 & 0.461 & 11.56 & 57.31 & 0.573 & 0.124 \\
\hline & Female & 26 & 119.54 & & & & 20.18 & & & \\
\hline PL & Male & 37 & 57.88 & 100.50 & 0.472 & 0.639 & 6.30 & 122.37 & 1.224 & 0.605 \\
\hline & Female & 26 & 57.59 & & & & 5.15 & & & \\
\hline MD & Male & 37 & 17.61 & 99.62 & -0.228 & 0.820 & 1.58 & 134.43 & 1.344 & 0.443 \\
\hline & Female & 26 & 17.67 & & & & 1.17 & & & \\
\hline CBL & Male & 37 & 116.53 & 99.83 & -0.221 & 0.826 & 10.31 & 72.41 & 0.724 & 0.369 \\
\hline & Female & 26 & 116.73 & & & & 14.23 & & & \\
\hline UT & Male & 37 & 44.99 & 101.35 & 1.180 & 0.245 & 2.46 & 49.09 & 0.491 & 0.050 \\
\hline & Female & 26 & 44.39 & & & & 5.01 & & & \\
\hline RL & Male & 37 & 57.47 & 101.06 & 1.072 & 0.290 & 2.48 & 38.39 & 0.384 & 0.009 \\
\hline & Female & 26 & 56.86 & & & & 6.46 & & & \\
\hline MRH & Male & 37 & 46.84 & 99.37 & -0.507 & 0.615 & 4.23 & 70.57 & 0.706 & 0.333 \\
\hline & Female & 26 & 47.13 & & & & 5.99 & & & \\
\hline $\mathrm{ACP}$ & Male & 37 & 36.84 & 99.17 & -0.628 & 0.532 & 4.21 & 125.04 & 1.250 & 0.565 \\
\hline & Female & 26 & 37.15 & & & & 3.37 & & & \\
\hline LT & Male & 37 & 52.62 & 100.92 & 1.005 & 0.320 & 2.65 & 68.61 & 0.686 & 0.300 \\
\hline & Female & 25 & 52.15 & & & & 3.86 & & & \\
\hline JT & Male & 37 & 6.43 & 95.63 & -2.217 & 0.031 & 0.22 & 72.79 & 0.728 & 0.377 \\
\hline & Female & 26 & 6.73 & & & & 0.30 & & & \\
\hline MH & Male & 37 & 12.43 & 95.32 & -1.899 & 0.064 & 1.06 & 54.43 & 0.544 & 0.093 \\
\hline & Female & 26 & 13.04 & & & & 1.94 & & & \\
\hline ML & Male & 37 & 86.60 & 99.11 & -0.701 & 0.487 & 12.28 & 52.27 & 0.523 & 0.074 \\
\hline & Female & 26 & 87.38 & & & & 23.50 & & & \\
\hline MW & Male & 37 & 21.88 & 97.53 & -1.188 & 0.240 & 5.54 & 315.11 & 3.151 & 0.004 \\
\hline & Female & 26 & 22.43 & & & & 1.76 & & & \\
\hline $\mathrm{i} 1 \mathrm{H}$ & Male & 36 & 4.03 & 107.93 & 1.995 & 0.051 & 0.34 & 105.30 & 1.053 & 0.906 \\
\hline & Female & 26 & 3.73 & & & & 0.32 & & & \\
\hline $\mathrm{i} 2 \mathrm{H}$ & Male & 36 & 4.29 & 106.34 & 1.400 & 0.167 & 0.53 & 107.95 & 1.080 & 0.854 \\
\hline & Female & 26 & 4.03 & & & & 0.49 & & & \\
\hline $\mathrm{i} 3 \mathrm{H}$ & Male & 37 & 5.11 & 103.14 & 0.786 & 0.435 & 0.62 & 105.54 & 1.055 & 0.902 \\
\hline & Female & 26 & 4.96 & & & & 0.59 & & & \\
\hline
\end{tabular}


Table 2. (Continued)

\begin{tabular}{|c|c|c|c|c|c|c|c|c|c|c|}
\hline \multirow{2}{*}{ Variable } & \multirow{2}{*}{ Sex } & \multirow{2}{*}{$\mathrm{N}$} & \multirow{2}{*}{ Mean } & \multirow{2}{*}{ Ratio (\%) } & \multicolumn{2}{|c|}{ Welch's $t$-test } & \multirow{2}{*}{ Variance } & \multirow{2}{*}{ Ratio (\%) } & \multicolumn{2}{|c|}{$F$-test } \\
\hline & & & & & $t$ & $P$ & & & $F$ & $P$ \\
\hline \multirow[t]{2}{*}{$\mathrm{cL}$} & Male & 37 & 5.54 & 103.59 & 1.649 & 0.105 & 0.18 & 77.98 & 0.780 & 0.486 \\
\hline & Female & 26 & 5.35 & & & & 0.23 & & & \\
\hline \multirow[t]{2}{*}{$\mathrm{cW}$} & Male & 37 & 4.01 & 108.50 & 3.605 & 0.001 & 0.16 & 176.78 & 1.768 & 0.140 \\
\hline & Female & 26 & 3.69 & & & & 0.09 & & & \\
\hline \multirow[t]{2}{*}{$\mathrm{p} 1 \mathrm{~L}$} & Male & 37 & 3.14 & 99.35 & -0.217 & 0.829 & 0.12 & 83.22 & 0.832 & 0.603 \\
\hline & Female & 26 & 3.17 & & & & 0.15 & & & \\
\hline \multirow[t]{2}{*}{$\mathrm{p} 2 \mathrm{~L}$} & Male & 36 & 5.54 & 99.01 & -0.689 & 0.495 & 0.06 & 50.65 & 0.507 & 0.063 \\
\hline & Female & 26 & 5.60 & & & & 0.12 & & & \\
\hline \multirow[t]{2}{*}{$\mathrm{p} 3 \mathrm{~L}$} & Male & 37 & 6.24 & 98.45 & -1.055 & 0.297 & 0.08 & 46.23 & 0.462 & 0.034 \\
\hline & Female & 26 & 6.34 & & & & 0.17 & & & \\
\hline \multirow[t]{2}{*}{$\mathrm{p} 4 \mathrm{~L}$} & Male & 37 & 7.49 & 100.13 & 0.107 & 0.915 & 0.12 & 88.35 & 0.884 & 0.721 \\
\hline & Female & 26 & 7.48 & & & & 0.14 & & & \\
\hline \multirow[t]{2}{*}{$\mathrm{p} 4 \mathrm{~W}$} & Male & 37 & 3.60 & 100.57 & 0.285 & 0.777 & 0.07 & 80.95 & 0.810 & 0.552 \\
\hline & Female & 26 & 3.58 & & & & 0.09 & & & \\
\hline $\mathrm{m} 1 \mathrm{~L}$ & Male & 37 & 12.64 & 101.33 & 1.240 & 0.221 & 0.24 & 82.64 & 0.826 & 0.590 \\
\hline & Female & 26 & 12.47 & & & & 0.30 & & & \\
\hline $\mathrm{m} 1 \mathrm{~W}$ & Male & 37 & 5.15 & 98.58 & -0.750 & 0.456 & 0.18 & 146.19 & 1.462 & 0.324 \\
\hline & Female & 26 & 5.23 & & & & 0.13 & & & \\
\hline $\mathrm{m} 2 \mathrm{~L}$ & Male & 36 & 6.56 & 97.35 & -1.934 & 0.058 & 0.13 & 100.25 & 1.002 & 0.989 \\
\hline & Female & 26 & 6.74 & & & & 0.13 & & & \\
\hline $\mathrm{m} 2 \mathrm{~W}$ & Male & 36 & 4.30 & 103.08 & 1.419 & 0.161 & 0.15 & 144.06 & 1.441 & 0.345 \\
\hline & Female & 26 & 4.17 & & & & 0.10 & & & \\
\hline $\mathrm{m} 3 \mathrm{~L}$ & Male & 37 & 3.05 & 93.55 & -2.119 & 0.038 & 0.18 & 144.35 & 1.444 & 0.349 \\
\hline & Female & 25 & 3.26 & & & & 0.12 & & & \\
\hline $\mathrm{m} 3 \mathrm{~W}$ & Male & 37 & 2.51 & 99.02 & -0.322 & 0.748 & 0.12 & 198.71 & 1.987 & 0.081 \\
\hline & Female & 25 & 2.53 & & & & 0.06 & & & \\
\hline $\mathrm{I} 1 \mathrm{H}$ & Male & 36 & 3.35 & 97.91 & -0.455 & 0.651 & 0.20 & 40.00 & 0.400 & 0.013 \\
\hline & Female & 26 & 3.42 & & & & 0.50 & & & \\
\hline $\mathrm{I} 2 \mathrm{H}$ & Male & 37 & 4.33 & 102.60 & 0.685 & 0.496 & 0.40 & 105.41 & 1.054 & 0.905 \\
\hline & Female & 26 & 4.22 & & & & 0.38 & & & \\
\hline $\mathrm{I} 3 \mathrm{H}$ & Male & 36 & 5.78 & 98.67 & -0.427 & 0.671 & 0.47 & 88.95 & 0.890 & 0.738 \\
\hline & Female & 26 & 5.86 & & & & 0.53 & & & \\
\hline CL & Male & 37 & 5.56 & 103.11 & 1.621 & 0.111 & 0.16 & 97.38 & 0.974 & 0.925 \\
\hline & Female & 26 & 5.39 & & & & 0.17 & & & \\
\hline $\mathrm{CW}$ & Male & 37 & 3.98 & 107.21 & 3.003 & 0.004 & 0.17 & 205.05 & 2.050 & 0.064 \\
\hline & Female & 26 & 3.71 & & & & 0.08 & & & \\
\hline P1L & Male & 37 & 3.49 & 96.78 & -1.527 & 0.133 & 0.09 & 111.33 & 1.113 & 0.790 \\
\hline & Female & 26 & 3.60 & & & & 0.08 & & & \\
\hline $\mathrm{P} 2 \mathrm{~L}$ & Male & 37 & 5.62 & 99.55 & -0.385 & 0.702 & 0.07 & 113.17 & 1.132 & 0.762 \\
\hline & Female & 25 & 5.65 & & & & 0.06 & & & \\
\hline P3L & Male & 37 & 6.73 & 99.62 & -0.246 & 0.807 & 0.16 & 93.97 & 0.940 & 0.849 \\
\hline & Female & 26 & 6.75 & & & & 0.17 & & & \\
\hline P4L & Male & 36 & 11.23 & 100.69 & 0.443 & 0.660 & 0.29 & 49.87 & 0.499 & 0.057 \\
\hline & Female & 26 & 11.16 & & & & 0.59 & & & \\
\hline P4W & Male & 36 & 6.00 & 97.36 & -1.279 & 0.206 & 0.30 & 141.81 & 1.418 & 0.366 \\
\hline & Female & 26 & 6.17 & & & & 0.21 & & & \\
\hline M1L & Male & 36 & 8.76 & 99.11 & -0.710 & 0.481 & 0.19 & 105.95 & 1.059 & 0.894 \\
\hline & Female & 26 & 8.84 & & & & 0.18 & & & \\
\hline M1W & Male & 37 & 9.19 & 101.17 & 0.777 & 0.440 & 0.26 & 84.43 & 0.844 & 0.631 \\
\hline & Female & 26 & 9.09 & & & & 0.30 & & & \\
\hline $\mathrm{M} 2 \mathrm{~L}$ & Male & 36 & 5.47 & 100.12 & 0.064 & 0.949 & 0.18 & 141.01 & 1.410 & 0.374 \\
\hline & Female & 26 & 5.47 & & & & 0.13 & & & \\
\hline M2W & Male & 37 & 6.16 & 99.97 & -0.018 & 0.986 & 0.24 & 132.76 & 1.328 & 0.463 \\
\hline & Female & 26 & 6.16 & & & & 0.18 & & & \\
\hline
\end{tabular}

Ratio: ratios of mean and variance, male/female $\times 100$ (\%). Bold: $P<0.05$. 
Table 3. The first 6 principal components which account for more than $80 \%$ of total variation from the sPCA

\begin{tabular}{|c|c|c|c|c|c|c|}
\hline Variable & $\mathrm{PC} 1$ & $\mathrm{PC} 2$ & PC3 & PC4 & PC5 & PC6 \\
\hline NPL & 0.754 & 0.128 & 0.239 & -0.382 & -0.295 & 0.264 \\
\hline NL & 0.684 & 0.187 & 0.298 & -0.386 & -0.308 & 0.341 \\
\hline FPL & 0.441 & -0.153 & -0.216 & 0.302 & 0.084 & -0.513 \\
\hline SH & 0.637 & 0.238 & -0.056 & 0.013 & 0.307 & -0.157 \\
\hline TL & 0.871 & 0.058 & 0.152 & -0.201 & -0.081 & -0.157 \\
\hline RB & 0.493 & 0.275 & -0.127 & 0.116 & 0.291 & 0.019 \\
\hline POC & -0.148 & 0.790 & -0.376 & 0.303 & -0.248 & 0.099 \\
\hline IC & 0.478 & 0.480 & -0.057 & 0.113 & 0.068 & -0.166 \\
\hline PB & 0.394 & 0.622 & -0.215 & 0.387 & -0.055 & 0.027 \\
\hline $\mathrm{OCB}$ & 0.247 & 0.106 & 0.070 & -0.091 & -0.130 & 0.200 \\
\hline ZB & 0.722 & 0.183 & -0.055 & 0.185 & 0.070 & -0.117 \\
\hline GL & 0.886 & 0.039 & 0.090 & -0.059 & -0.141 & -0.209 \\
\hline PL & 0.665 & 0.228 & 0.140 & -0.250 & -0.228 & -0.161 \\
\hline MD & 0.413 & 0.346 & 0.020 & -0.378 & 0.518 & -0.311 \\
\hline CBL & 0.787 & 0.081 & 0.174 & -0.196 & -0.103 & -0.134 \\
\hline UT & 0.699 & 0.250 & -0.025 & -0.345 & -0.219 & -0.282 \\
\hline RL & 0.723 & 0.246 & 0.007 & -0.371 & -0.146 & -0.237 \\
\hline MRH & 0.775 & -0.152 & -0.182 & 0.146 & 0.145 & -0.128 \\
\hline $\mathrm{ACP}$ & 0.703 & 0.070 & 0.113 & 0.166 & 0.289 & -0.128 \\
\hline LT & 0.706 & 0.121 & -0.160 & -0.273 & -0.213 & -0.320 \\
\hline JT & 0.557 & -0.102 & -0.468 & -0.055 & 0.418 & 0.491 \\
\hline MH & 0.710 & -0.448 & -0.348 & 0.194 & -0.259 & -0.078 \\
\hline ML & 0.896 & -0.165 & -0.034 & 0.082 & -0.113 & 0.012 \\
\hline MW & 0.501 & 0.005 & 0.691 & 0.476 & 0.110 & 0.122 \\
\hline Eigenvalue & 0.026 & 0.009 & 0.008 & 0.006 & 0.005 & 0.004 \\
\hline Proportion & 36.639 & 12.456 & 11.281 & 8.270 & 7.140 & 5.897 \\
\hline Cumulative & 36.639 & 49.094 & 60.375 & 68.645 & 75.786 & 81.682 \\
\hline
\end{tabular}

Bold: absolute value $>0.5$.

were canine widths, followed by length of $\mathrm{m} 3$, jaw thickness and breadth of postorbital constriction. These results differ from that of Haba et al. [5], which reported significant differences in CBL and upper canine length among the specimens from Hokkaido, as well as differences in lower canine width, length of $\mathrm{m} 3$, upper canine length and width of M1 among specimens from Honshu.

It is known that species with prominently sexually dimorphic canines often have a polygynous mating system, and canines are often recruited for male-male competitions [3]. On the other hand, those with a more relaxed sexual dimorphism in the canines tend to exhibit monogamous mating systems [4]. The raccoon dog is reported to be monogamous in Korea, Japan and Finland [2, 8, 9, 23], although the mating systems of the species have not been studied in other populations. Future field observations of the interactions between males, especially on the use of canines for inter-male competitions or associated behavior, should shed further light on the evolution of arrested sexual dimorphism in the raccoon dogs.

The number and kinds of sexually dimorphic traits seem to be different between populations, as has been shown in our study as well as further previous studies [5, 16]. One explanation for this variability is that the selective forces acting upon the craniodental morphology of each sex may be different between populations. However, it should be noted that many craniodental traits are often associated with one another [11, 18, 19]. If quantitative aspects of such traits are significantly integrated in a likewise manner, the apparent sexual dimorphism observed in some traits might be a byproduct of true sexual dimorphism. To investigate this possibility, geographically broader studies using comparable data from many distinct populations are required.

CVA using skull variables discriminated between the sexes more clearly than tCVA, despite using fewer skull variables than those of teeth. Scores of sPC2 and those of tPC4 were significantly different between sexes, but the total variation which we could account for was higher in the former. These results indicate that the overall sexual difference is more apparent from data relating to the skull than that relating to the teeth. In general, tooth measurements are more variable than skull measurements within a given population of carnivoran species $[19,21]$. Therefore, although the clearest differences are found among canine widths, it is likely that the highly variable nature of the teeth may obscure the discrimination between sexes.

ACKNOWLEDGMENTS. We express our gratitude to everyone who donated samples to Conservation Genome Resource Bank for Korean Wildlife. We also thank Dr. Su-Yuan SEO of Ewha Womans University for providing skull specimen. This work was supported by the National 
Table 4. The first 7 principal components which account for more than $80 \%$ of total variation from the tPCA

\begin{tabular}{|c|c|c|c|c|c|c|c|}
\hline Variable & PC1 & $\mathrm{PC} 2$ & PC3 & PC4 & PC5 & PC6 & PC7 \\
\hline $\mathrm{i} 1 \mathrm{H}$ & 0.811 & -0.114 & -0.135 & -0.432 & -0.042 & 0.129 & 0.036 \\
\hline $\mathrm{i} 2 \mathrm{H}$ & 0.874 & -0.100 & -0.167 & -0.229 & -0.183 & 0.081 & -0.132 \\
\hline $\mathrm{i} 3 \mathrm{H}$ & 0.904 & -0.112 & 0.055 & 0.018 & 0.060 & 0.081 & 0.067 \\
\hline $\mathrm{cL}$ & 0.198 & 0.579 & 0.400 & -0.241 & 0.078 & -0.182 & -0.396 \\
\hline $\mathrm{cW}$ & 0.131 & 0.698 & 0.281 & -0.228 & 0.269 & -0.197 & -0.031 \\
\hline $\mathrm{p} 1 \mathrm{~L}$ & 0.244 & 0.535 & 0.098 & 0.046 & -0.635 & -0.044 & 0.216 \\
\hline p2L & 0.177 & 0.569 & 0.192 & 0.198 & -0.144 & 0.038 & -0.122 \\
\hline p3L & 0.075 & 0.428 & 0.045 & 0.239 & -0.286 & 0.285 & -0.132 \\
\hline $\mathrm{p} 4 \mathrm{~L}$ & 0.106 & 0.663 & 0.017 & 0.053 & -0.097 & 0.190 & 0.220 \\
\hline $\mathrm{p} 4 \mathrm{~W}$ & 0.229 & 0.683 & -0.045 & -0.025 & 0.338 & 0.218 & 0.111 \\
\hline $\mathrm{m} 1 \mathrm{~L}$ & 0.248 & 0.709 & 0.137 & 0.036 & -0.146 & 0.204 & 0.029 \\
\hline $\mathrm{m} 1 \mathrm{~W}$ & 0.201 & 0.805 & 0.009 & 0.082 & 0.065 & 0.139 & 0.172 \\
\hline $\mathrm{m} 2 \mathrm{~L}$ & 0.047 & 0.333 & -0.129 & 0.394 & 0.211 & 0.254 & 0.021 \\
\hline $\mathrm{m} 2 \mathrm{~W}$ & 0.219 & 0.289 & -0.226 & -0.121 & 0.609 & 0.372 & -0.068 \\
\hline $\mathrm{m} 3 \mathrm{~L}$ & -0.042 & 0.244 & -0.878 & 0.150 & -0.094 & -0.192 & 0.009 \\
\hline $\mathrm{m} 3 \mathrm{~W}$ & 0.157 & 0.328 & -0.769 & 0.157 & 0.172 & -0.250 & -0.142 \\
\hline $\mathrm{I} 1 \mathrm{H}$ & 0.699 & -0.086 & 0.131 & 0.624 & 0.002 & 0.198 & -0.128 \\
\hline $\mathrm{I} 2 \mathrm{H}$ & 0.748 & -0.203 & 0.055 & 0.115 & 0.330 & -0.300 & 0.367 \\
\hline $\mathrm{I} 3 \mathrm{H}$ & 0.548 & 0.009 & 0.250 & 0.241 & -0.160 & -0.602 & -0.146 \\
\hline CL & 0.291 & 0.514 & 0.432 & -0.234 & 0.165 & -0.152 & -0.364 \\
\hline $\mathrm{CW}$ & 0.292 & 0.672 & 0.276 & -0.275 & 0.268 & -0.208 & 0.065 \\
\hline P1L & 0.157 & 0.551 & -0.031 & -0.149 & -0.413 & -0.031 & 0.111 \\
\hline $\mathrm{P} 2 \mathrm{~L}$ & -0.012 & 0.573 & 0.298 & 0.060 & -0.009 & 0.167 & 0.188 \\
\hline P3L & -0.083 & 0.314 & 0.139 & 0.330 & -0.089 & 0.173 & 0.025 \\
\hline P4L & 0.044 & 0.743 & 0.139 & 0.179 & -0.083 & 0.108 & 0.015 \\
\hline P4W & 0.116 & 0.719 & -0.086 & 0.170 & -0.051 & -0.021 & 0.096 \\
\hline M1L & 0.115 & 0.648 & 0.071 & 0.174 & -0.199 & 0.289 & 0.315 \\
\hline M1W & 0.176 & 0.763 & 0.106 & 0.033 & 0.144 & 0.175 & 0.014 \\
\hline M2L & 0.040 & 0.380 & -0.163 & 0.173 & 0.017 & 0.441 & 0.302 \\
\hline M2W & 0.207 & 0.463 & -0.089 & 0.074 & 0.388 & 0.192 & -0.291 \\
\hline Eigenvalue & 0.097 & 0.046 & 0.030 & 0.024 & 0.019 & 0.017 & 0.010 \\
\hline Proportion & 32.084 & 15.403 & 10.019 & 7.981 & 6.223 & 5.511 & 3.155 \\
\hline Cumulative & 32.084 & 47.487 & 57.506 & 65.487 & 71.709 & 77.220 & 80.375 \\
\hline
\end{tabular}

Bold: absolute value $>0.5$.

Research Foundation of Korea (NRF) grant funded by the Korean government (MEST) 2009-0085754.

\section{REFERENCES}

1. Ansorge, H., Ranyuk, M., Kauhala, K., Kowalczyk, R. and Stier, N. 2009. Raccoon dog, Nyctereutes procyonoides, populations in the area of origin and in colonized regions - the epigenetic variability of an immigrant. Ann. Zool. Fenn. 46: 51-62.

2. Choi, T. Y. and Park, C. H. 2006. Home-range of raccoon dog Nyctereutes procyonoides living in the rural area of Korea. $J$. Ecol. Field Biol. 29: 259-263 (in Korean with English summary). [CrossRef]

3. De Marinis, A. M. 1995. Craniometric variability of polecat Mustela putorius L. 1758 from North-Central Italy. Hystrix 7: 57-68.

4. Gittleman, J. L. and Van Valkenburgh, B. 1997. Sexual dimorphism in the canines and skulls of carnivores: effects of size, phylogeny, and behavioural ecology. J. Zool. (Lond.) 242: 97-117. [CrossRef]

5. Haba, C., Oshida, T., Sasaki, M., Endo, H., Ichikawa, H. and Masuda, Y. 2008. Morphological variation of the Japanese rac- coon dog: implications for geographical isolation and environmental adaptation. J. Zool. (Lond.) 274: 239-247. [CrossRef]

6. Heptner, V. G. and Naumov, N. P. 1967. Mlekopitayushchie Sovetskogo Soyuza, Vysshaya Shkola Publisherss, Moscow (in Russian).

7. Hidaka, S., Matsumoto, M., Hiji, H., Ohsako, S. and Nishida, H. 1998. Morphology and morphometry of skulls of raccoon dogs, Nyctereutes procyonoides and badgers, Meles meles. J. Vet. Med. Sci. 60: 161-167. [Medline] [CrossRef]

8. Ikeda, H. 1982. Socio-ecological study on the raccoon dog, Nyctereutes procyonoides viverrinus, with reference to the habitat utilization pattern. Ph.D. Thesis, Kyushu University.

9. Kauhala, K., Helle, E. and Taskinen, K. 1993. Home range of the raccoon dog (Nyctereutes procyonoides) in southern Finland. $J$. Zool. (Lond.) 231: 95-106. [CrossRef]

10. Kauhala, K., Viranta, S., Kishimoto, M., Helle, E. and Obara, I. 1998. Comparison of skull morphology of Finnish and Japanese raccoon dogs. Ann. Zool. Fenn. 35: 1-16.

11. Koyabu, D., Endo, H., Mitgutsch, C., Suwa, G., Catania, K. C., Zollikofer, C. P. E., Oda, S., Koyasu, K., Ando, M. and SánchezVillagra, M. R. 2011. Heterochrony and developmental modularity of cranial osteogenesis in lipotyphlan mammals. Evodevo 2 : 
21. [Medline] [CrossRef]

12. Mazák, J. 2008. Craniometric variation in the tiger (Panthera tigris): implications for patterns of diversity, taxonomy and conservation. Mamm. Biol. 75: 45-68.

13. Miyao, T. and Koike, Y. 1982. Regional size-differences of skulls and teeth of raccoon dog, Nyctereutes procyonoides, collected in Yashiro Island and northern Kyushu. J. Growth 21: 69-79 (in Japanese with English abstract).

14. Monakhov, V. G. 2009. Is sexual size dimorphism variable? Data on species of the genus Martes in the Urals. Biol. Bull. 36: 45-52. [CrossRef]

15. Mori, T. 1922. On some new mammals from Korea and Manchuria. Ann. Mag. Nat. Hist. Zool. Bot. Geol. Ser. 9 10: 607-614.

16. Natori, M., Sakurai, H. and Shigahara, N. 2001. Geographical variation of sexual dimorphism in cranial measurements of Japanese raccoon dogs. J. Growth 40: 83-89.

17. Obara, I., Oishi, N. and Tsuchiya, K. 2006. Geographic variation in the mandible of raccoon dog, Nyctereutes procyonoides - an attempt to apply multivariate morphometric analysis-. Animate 6: 5-11 (in Japanese with English summary).

18. Porto, A., de Oliveira, F. B., Shirai, L. T., De Conto, V. and
Marroig, G. 2009. The Evolution of modularity in the mammalian skull I: morphological integration patterns and magnitudes. Evol. Biol. 36: 118-135. [CrossRef]

19. Prevosti, F. J. and Lamas, L. 2006. Variation of cranial and dental measurements and dental correlations in the pampean fox (Dusicyon gymnocercus). J. Zool. (Lond.) 270: 636-649. [CrossRef]

20. R Development Core Team, R Foundation for Statistical Computing. 2011. R: A language and environment for statistical computing [cited 2011 October 2], Available from. http://www.rproject.org/.

21. Suzuki, S., Abe, M. and Motokawa, M. 2012. Integrative study on static skull variation in the Japanese weasel (Carnivora: Mustelidae). J. Zool. (Lond.) 288: 57-65. [CrossRef]

22. Takeuchi, M. 2010. Sexual dimorphism and relative growth of body size in the Japanese red fox Vulpes vulpes japonica. Mammal Study 35: 125-131. [CrossRef]

23. Yashiki, H. 1987. Ecological study on the raccoon dog, Nyctereutes procyonoides viverrinus, in Shiga Heights. Bull. Inst. Nat. Educ. Shiga Heights Shinshu Univ. 24: 43-53 (in Japanese with English summary). 